\title{
Definições de zona de manejo para soja de alta produtividade
}

\section{Management zone settings for high productivity soybean}

\author{
Anderson Da Silva Umbelino ${ }^{\star}$, Danilo Gomes De Oliveira², Marcos Paulo De Oliveira Martins ${ }^{1}$ \\ e Elton Fialho Dos Reis ${ }^{1}$
}

\author{
1 Universidade Estadual de Goiás - UEG, Br 153, N03105 Fazenda Barreiro do Meio-Campus Henrique Santillo-Anápolis, Anápolis, G0, Brasil \\ ${ }_{2}^{2}$ Instituto Federal de Goiás - IFG, Rua Correntina, 824 - St. Dom Prudencio, Posse - G0, CEP - 73900-000, Posse, G0, Brasil \\ ( ${ }^{*}$-mail: anderson-umbelino@hotmail.com) \\ http://dx.doi.org/10.19084/RCA18092
}

Recebido/received: 2018.04.04

Recebido em versão revista/received in revised form: 2018.06.10

Aceite/accepted: 2018.06 .11

\section{R E S U M O}

O conhecimento dos padrões espaciais de parâmetros químicos dos solos são informações de suma importância para agricultura de precisão. Objetivou-se com este trabalho definir zonas de manejo por meio de classes de atributos químicos e produtividade média de soja em um campo de alta produção. As produtividades da soja foram quantificadas por meio de plantas coletadas em $1 \mathrm{~m}^{2}$, e os atributos químicos por meio de cinco coletas simples por ponto na camada de 0,00-0,20m num grid de 88 pontos de $60 \mathrm{~m} \times 60 \mathrm{~m}$ em dois anos consecutivos, sendo a massa de grãos pesadas e extrapoladas para t ha-1 considerando umidade de 13\%. Os mapas de classes foram gerados pelo software GS+ versão 7.0 em intervalos da média +/- 0,5 vezes o desvio padrão. A área de cada classe de produtividade foi calculada com o auxílio do software Google Earth por meio de sobreposição dos mapas. A classe considerada alta para produtividade foi predominante na região noroeste do mapa apresentando 8,217 ha, 6,508 ha de alto teor de $\mathrm{K}$, 4,769 ha de alto teor de CTC e 3,708 ha de alto teor de M.O. Mesmo não apresentado correlação significativa a produtividade e atributos químicos apresentaram maiores valores na região noroeste do mapa.

Palavras-chave: classes de produtividade, dependência espacial, geoestatística

\begin{abstract}
A B S T R A C T
Knowledge of spatial patterns of soil chemical parameters is critical information for precision agriculture. The objective of this work was to define management zones by means of classes of chemical attributes and average yield of soybean in a field of high production. Soybean yields were quantified by means of plants collected in $1 \mathrm{~m} 2$ and the chemical attributes by means of five single samples per point in the layer of $0.00-0.20 \mathrm{~m}$ in a grid of 88 points of $60 \mathrm{mX60m}$ in two consecutive years, mass of heavy grains and extrapolated for $\mathrm{t}$ ha- 1 considering $13 \%$ humidity. The class maps were generated by GS + version 7.0 software in average intervals +/- 0.5 times the standard deviation. The area of each productivity class was calculated with the help of the Google Earth program through overlapping maps. The class considered high for productivity was predominant in the northwest region of the map presenting 8,217 ha, 6,508 ha of high K content, 4,769 ha of high CTC content and 3,708 ha of high M.O. Although no significant correlation was found, productivity and chemical attributes presented higher values in the northwest region of the map.
\end{abstract}

Keywords: productivity classes, spatial dependency, geostatistics

\section{INTRODUÇÃO}

Caracterizada pela elevada quantidade de informações disponibilizadas, a aplicação da geoestatística na agricultura de precisão tem por finalidade caracterizar a variabilidade espacial dos atributos do solo e da cultura, estimando as inter-relações desses atributos no espaço e no tempo (Nascimento 
et al., 2014). Vários estudos têm buscado elucidar a variabilidade espacial de características químicas do solo e seu possível efeito sobre a produtividade das culturas (Mattioni et al., 2013).

O estudo da variabilidade espacial dos atributos do solo similarmente assume importância, nos indicativos de alternativas de manejo, onde o crescimento e desenvolvimento das plantas podem ser influenciados por alterações nos atributos químicos do solo (Montanari et al., 2015), e a produtividade das culturas pode ser variável em uma mesma área por menor que seja o grau de variação espacial de determinados atributos (Matias et al., 2015).

Apesar de representar o potencial produtivo de uma determinada área, a produtividade não é homogênea, sendo notado em um mesmo campo de produção regiões com maior e menor desempenho produtivo (Bottega et al., 2013a).

Zonas de manejo são os resultados de um planejamento adequado de uso e ocupação do solo, sendo áreas passíveis de receber as mesmas práticas agronômicas, vindo a reduzir os impactos do homem sobre o meio ambiente (Miqueloni et al., 2015).

A geoestatística constitui a base para a aplicação dos conceitos de agricultura de precisão, com análise da variabilidade espacial e temporal dos fatores inerentes à produtividade agrícola (Nascimento et al., 2014). O estudo da variabilidade espacial por meio da geoestatística possibilita a interpretação dos resultados com base na estrutura da variabilidade natural dos atributos avaliados, considerando a dependência espacial dentro do intervalo de amostragem (Souza et al., 2009). Essa ferramenta permite identificar zonas com restrições químicas que possam estar limitando o rendimento e, posteriormente, realizar a correção de maneira adequada (Matias et al., 2015).

No âmbito da geoestatística, o estudo da variabilidade espacial dos atributos físicos do solo utilizando a geoestatística, permite por meio desta análise, detectar a variabilidade e distribuição espacial dos atributos estudados e, portanto, constitui importante ferramenta na análise e descrição detalhada da variabilidade dos atributos do solo (Alves et al., 2014).
Objetivou-se com este trabalho definir zonas de manejo por meio de classes de atributos químicos e produtividade média de soja em um campo de alta produção.

\section{MATERIAL E MÉTODOS}

O estudo foi conduzido em área comercial na Fazenda Madeira localizada no município de Gameleira de Goiás, GO, com altitude de 940 m, e coordenadas geográficas $16^{\circ} 22^{\prime} 17.3^{\prime \prime}$ de latitude sul e $48^{\circ} 35^{\prime} 20.5^{\prime \prime}$ de longitude oeste. Foi utilizada uma área de pivô central com 36 hectares, que apresenta Latossolo Vermelho (EMBRAPA, 2013) com textura argilosa e topografia com pouca declividade, georreferenciado por meio de uma malha amostral sistemática, utilizando um receptor de GPS Garmim Etrex trabalhando no sistema WGS 84, ajustado com intervalo de $60 \mathrm{~m}$ entre pontos de amostragem, totalizando 88 pontos (Figura 1). Foram coletadas em torno de cada ponto do grid amostral, amostras deformadas do solo na camada de 0,00-0,20 m, composta de cinco amostras simples, coletadas num raio de 5 metros, posteriormente foram embaladas, vedadas encaminhadas para um laboratório de solos comercial para determinação dos atributos químicos do solo, segundo metodologias propostas por Donagema et al. (2011) onde foram determinados, Potencial Hidrogeniônico - $(\mathrm{pH})$, potássio $-\left(\mathrm{K}^{+}\right)$, fósforo disponíveis $\left(\mathrm{P}, \mathrm{Mehlich}^{-1}\right)$ e teor em matéria orgânica (M.O.). Posteriormente foi calculada a capacidade de troca cationica (CTC). Os dados de produtividade foram obtidos por meio de plantas coletadas em um metro quadrado, sendo a massa de grãos pesada e extrapolada para $\mathrm{t} \mathrm{ha}^{-1}$ considerando umidade de $13 \%$ b.u. (Weber, 2001) nos diferentes pontos amostrados. A área possui histórico de mapeamento de dois anos representativos das safras de 2014/2015 e 2015/2016 propiciando subsídio para a produtividade média dos dois anos consecutivos. Após esta análise, confeccionou-se o mapa da média das duas safras por meio da Eq. 1:

$$
\overline{\mathrm{P}} \mathrm{l}=\sum_{\mathrm{j}=1}^{\mathrm{r}} \frac{\mathrm{Pij}}{\mathrm{r}}
$$


Em que:

$\mathrm{Pi}=$ Produtividade média de soja no ponto $\mathrm{i}$, do mapa interpolado, nas duas safras estudadas;

$P i j=$ Produtividade no ponto i na safra $j ;$

$\mathrm{r}=$ número de safras estudadas.

Foi utilizada a estatística descritiva para verificar a existência de tendência central e caracterizar a dispersão dos dados. A filtragem dos dados foi realizada com auxílio dos gráficos box-plot, que foram construídos a fim de identificar valores discrepantes (outliers). Foi calculada uma matriz de correlação de Pearson a nível de 5\% de probabilidade, entre as características químicas do solo e a produtividade, permitindo uma análise de relação entre os mesmos. Todavia Montgomery e Runger (2009) enfatizam que o número de pontos utilizados na análise de correlação influência de forma tendenciosa o teste de hipótese, quanto maior o número de pontos, maior a chance de rejeição da hipótese de nulidade. Os autores recomendam a utilização de 30 pontos para análise de correlação. Com isso foram retiradas 30 amostras aleatórias do conjunto de dados para realização da análise. A normalidade dos dados foi avaliada aplicando o teste de Shapiro e Wilk (1965) a nível de 5\% de probabilidade. Estas análises foram realizadas em planilha eletrônica.

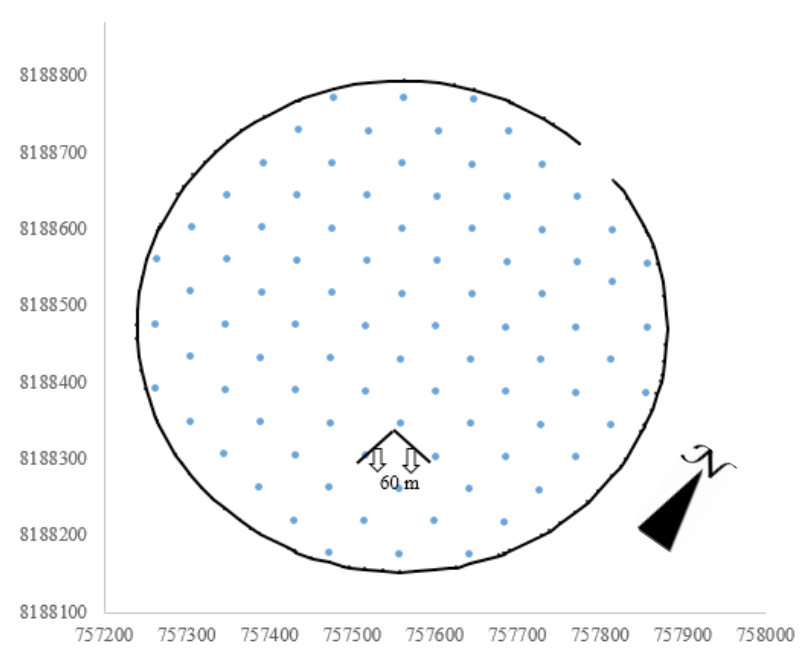

Figura 1 - Croqui da malha sistemática amostral na área de estudo.
Para identificar e quantificar a dependência espacial, utilizou-se modelagem e análise variográfica, ajustando aos valores de semivariância empírica, modelos teóricos de semivariância. Após este procedimento, construiu-se os semivariogramas experimentais utilizando o software computacional GS+, versão 7.0 (Gamma Design Software ${ }^{\circledR}$ ). Os modelos testados foram o Gaussiano, Esférico, Exponencial e Linear com patamar. Selecionou-se aquele que apresentou maior coeficiente de determinação $\left(R^{2}\right)$ e menor soma de quadrados de resíduos (RSS) (Matias et al., 2015). A classificação e a determinação do Índice de Dependência Espacial (IDE) foi de acordo com Zimback (2001) utilizando a equação 2 e assumindo os seguintes intervalos: dependência espacial baixa para IDE $<25 \%$, moderada para $25 \%<\mathrm{IDE}<75 \%$ e forte para IDE $>75 \%$.

$\mathrm{IDE}=\frac{\mathrm{C}_{0}}{\mathrm{C}_{0}+\mathrm{C}} 100$

Em que:

IDE = índice de dependência espacial;

$\mathrm{C}=$ contribuição da semivariância $\left(\mathrm{C}_{0}+\mathrm{C}-\mathrm{C}_{0}\right)$;

$\mathrm{C}_{0}+\mathrm{C}=$ patamar

A elaboração dos mapas temáticos de distribuição espacial, incluindo o mapa da média de produtividade e atributos químicos foram gerados com o auxílio do software geoestatístico. Foram confeccionados mapas de classes de produtividade (alta, média e baixa) para cada safra, utilizando os parâmetros de produtividade do ponto com valor acima da média do talhão mais 0,5 vezes o desvio padrão (produtividade alta); produtividade do ponto com valor compreendido no intervalo entre a média do talhão mais 0,5 vezes o desvio padrão e a média do talhão menos 0,5 vezes o desvio padrão (produtividade média); produtividade do ponto com valor abaixo da média do talhão menos 0,5 vezes o desvio padrão (produtividade baixa), adaptado de Bottega et al., (2013b). Também foram confeccionados mapas de classes (alto, médio e baixo) dos atributos químicos utilizando o mesmo procedimento. Os mapas de classes foram gerados pelo software GS+ versão 7.0 e as áreas de cada classe foram calculadas com o auxílio do software Google Earth por meio de sobreposição dos mapas. 


\section{RESULTADOS E DISCUSSÃO}

A fim de verificar os parâmetros de tendência central e dispersão, foi realizada uma análise descritiva, utilizadas como ferramenta auxiliar, para complementar o comportamento das variáveis estudadas. $\mathrm{O}$ teste de normalidade de Shapiro \& Wilk (1965) evidenciou normalidade para $\mathrm{P}, \mathrm{pH}$ e M.O., mas não para os dados de produtividade, $\mathrm{Ke}$ CTC, ao nível de $5 \%$ de probabilidade.

Os valores dos coeficientes de variação apresentaram-se como altos $(20 \%<\mathrm{CV}<30 \%)$ para a produtividade, médios $(10 \%<\mathrm{CV}<20 \%)$ para $\mathrm{K}, \mathrm{CTC}$, M.O. e baixo $(\mathrm{CV}<10 \%)$ para o $\mathrm{PH}$, conforme Pimentel Gomes e Garcia (2002). O pH do solo apresentou média de 5,47 e desvio padrão de 0,20 , mostrando uma satisfatória repartição dos pontos com relação ao $\mathrm{pH}$, já que o $\mathrm{CV}$ apresentou baixo $(3,57)$, expressando o desvio padrão em relação à média e a significância do teste de normalidade para o $\mathrm{pH}$. A média apresenta valor de $\mathrm{pH}$ considerado dentro da faixa de acidez, faixa esta onde a maioria dos nutrientes está disponível para absorção pelas plantas, sendo o ideal por volta de 5,5 a 6 (Artur et al., 2014).

Observou-se também tendência de concentração dos valores de $\mathrm{pH}$ pouco abaixo da média apresentando uma assimetria negativa. No entanto as condições do solo em estudo exibiram fracas correlações negativas não significativas a $5 \%$ de probabilidade entre o $\mathrm{pH}$ e $\mathrm{P}$ do mesmo modo para $\mathrm{pH}$ e M.O., consistindo em - 0,02 para fósforo e $-0,12$ para matéria orgânica.

A M.O. é o principal contribuinte para o aumento da CTC do solo, sobretudo, em solos de regiões tropicais altamente intemperizados como os Latossolos (MATIAS et al., 2015). No entanto esses dois atributos apresentaram baixo valor de correlação não significativo a $5 \%$ de probabilidade $(0,14)$.

O nível de $\mathrm{P}$ no solo apresentou valores expressivos com média de $99,75 \mathrm{mg} \mathrm{dm}^{-3}$ indicando sua suficiência para a produtividade da soja, visto que, o P é considerado um nutriente importante na nutrição das plantas, sendo recomendada a fosfatagem em casos em que há deficiência de $\mathrm{P}\left(\mathrm{P}<8.0 \mathrm{mg} / \mathrm{dm}^{3}\right)$. Quanto à dispersão relativa dos dados, fornecida pelo CV $(97,51)$, constatou-se que os teores de $\mathrm{P}$ apresentaram $\mathrm{CV}$ classificado como muito alto, desigualmente dos resultados de Cherubin et al. (2015) em que encontraram CV moderados e altos para caracterização da variabilidade espacial de fósforo em Latossolo Vermelho em diferentes malhas amostrais.

A área apresentou elevada produtividade, com uma média de 5,07 $\mathrm{t} \mathrm{ha}^{-1}$, tendo uma variação de 3,27 tha-1 a 6,69 t ha-1 (Quadro 1), apresentando um CV classificado como alto $(24,80 \%)$, sendo a distribuição espacial ilustrada na Figura 2. Mattioni et al. (2013), estudaram a variabilidade espacial e efeito de atributos químicos de um Latossolo na produtividade de grãos de soja, depararam com produtividade média de $3.54 \mathrm{t} \mathrm{ha}^{-1} \mathrm{e}$ variabilidade média expressa por um coeficiente de variação de $9,66 \%$, contudo, Bottega et al. (2013), encontraram uma produtividade da média de 3 anos consecutivos de $4,17 \mathrm{t} \mathrm{ha}^{-1}$ expressa por $\mathrm{CV}$ de $9,7 \%$.

O modelo matemático que melhor se ajustou aos semivariogramas foi o exponencial para produtividade média, potássio, fósforo e Capacidade de Troca Catiônica e modelo, já para o pH ajustou-se

Quadro 1 - Estatística descritiva para produtividade média $(P R)$, fósforo $(P)$; potássio $(K)$; capacidade de trocas catiônicas (CTC) potencial Hidrogeniônico (pH), e matéria orgânica (MO)

\begin{tabular}{|c|c|c|c|c|c|c|}
\hline Parâmetros & PR & $\mathbf{P}$ & $\mathbf{K}$ & CTC & PH & MO \\
\hline Estatísticos & $\left(\mathrm{t} \mathrm{h}^{-1}\right)$ & $\begin{array}{c}(\mathrm{mg} \\
\left.\mathrm{dm}^{-3}\right)\end{array}$ & $\begin{array}{c}(\mathrm{mg} \\
\left.\mathrm{dm}^{-3}\right)\end{array}$ & $\begin{array}{c}\left(\mathrm{cmol}_{\mathrm{c}}\right. \\
\left.\mathrm{dm}^{-3}\right)\end{array}$ & $\left(\mathrm{CaCl}_{2}\right)$ & $(\%)$ \\
\hline Média & 5,07 & 99,75 & 247,36 & 7,66 & 5,47 & 1,64 \\
\hline Mínimo & 3,23 & 14,00 & 176 & 5,20 & 4,90 & 1,20 \\
\hline Máximo & 6,69 & 390,00 & 320 & 10,90 & 5,90 & 2,30 \\
\hline Mediana & 5,00 & 47,00 & 242 & 7,65 & 5,50 & 1,60 \\
\hline Assimetria & 0,14 & 1,16 & 0,99 & 0,39 & $-0,32$ & 0,91 \\
\hline Curtose & 0,34 & 0,07 & $-0,42$ & 0,65 & 0,78 & 2,90 \\
\hline D. Padrão & 0,64 & 97,27 & 34,78 & 1,03 & 0,20 & 0,18 \\
\hline Variância & 0,41 & 9461,95 & 1209,70 & 1,06 & 0,04 & 0,03 \\
\hline Teste W & $0,984^{\mathrm{ns}}$ & $0,77^{*}$ & $0,981^{\mathrm{ns}}$ & $0,983^{\text {ns }}$ & $0,942^{*}$ & $0,598^{*}$ \\
\hline C.V. (\%) & 24,80 & 97,51 & 14,40 & 13,44 & 3,57 & 11,06 \\
\hline C. PER. & 1,000 & $0,340^{\mathrm{ns}}$ & $0,235^{\text {ns }}$ & $0,183^{\mathrm{ns}}$ & $-0,346^{\text {ns }}$ & $0,340^{\mathrm{n}}$ \\
\hline
\end{tabular}

CV: coeficiente de variação; CP: coeficiente de correlação de Pearson em relação à produtividade de massa verde $\left(^{\star}\right)$ significativo à $5 \%$ de probabilidade; (ns) não significativo à $5 \%$ de probabilidade; Teste W: teste de normalidade de Shapiro \& Wilk; $\left(^{*}\right)$ significativo ao teste de normalidade de Shapiro \& Wilk à $5 \%$ de probabilidade; (ns) não significativo ao teste de normalidade de Shapiro \& Wilk à $5 \%$ de probabilidade. 
o modelo gaussiano. Estudos relacionados com a variabilidade espacial de atributos do solo têm mostrado no geral que o modelo esférico e exponencial tem sido como os de maior ocorrência (Alves et al., 2014; Aquino et al., 2014; Matias et al., 2015; Cherubin et al., 2015), corroborando assim com os modelos ajustados no presente estudo (Quadro 2).

Alcances expostos pelos semivariogramas ajustados para os atributos químicos do solo mostraram valores entre 63 e $481 \mathrm{~m}$, contudo a análise dos semivariogramas permite deduzir que a malha sistemática utilizada para a amostragem foi eficiente para obter dados representativos da área, pois nenhum dos alcances foi menor do que a menor distância entre os pontos amostrados, exceto para M.O. que apresentou efeito pepita puro, que é um importante parâmetro do semivariograma, e indica variabilidade não explicada, considerando a distância de amostragem utilizada na área e se sua variabilidade é aleatória (Ferraz et al., 2017). O semivariograma avalia a dependência espacial, como não ocorreu a dependência espacial, destaca-se que neste caso não se deve empregar o método geoestatística para variável (Reis et al., 2012), deve ser utilizado o valor médio. Nesse estudo, a relação $\mathrm{C} 0 / \mathrm{C} 0+\mathrm{C} 1$ (equação 2) mostrou dependência espacial moderada para todos os atributos com exceção para CTC e pH, que obtiveram forte dependência, segundo a classificação de Zimback (2001).

O maior coeficiente de determinação observado foi 0,979 para potássio. Bottega et al. (2013a) ressaltaram que somente este valor não é indicativo de que o ajuste obtido é o que melhor representa a variabilidade espacial do atributo em estudo, e que este coeficiente pode ser utilizado como parâmetro na decisão de qual modelo melhor se ajustou a semivariância experimental.

A análise da variabilidade espacial dos atributos químicos e produtividade apresentaram dependência espacial, o que possibilita a estimativa dos valores nos locais não amostrados por meio do interpolador geoestatístico da krigagem (Cherubin et al., 2015), depois de detectada variabilidade espacial, utilizou-se o método de interpolação para confecção dos mapas temáticos de distribuição espacial. A construção dos mapas de krigagem, obtidos por meio dos ajustes do semivariogramas, permitiu identificar e delimitar área com maior e menor variabilidade do solo, possibilitando aplicações de insumos (corretivos e fertilizantes) com taxa variável, visando à sua implementação em quantidades adequadas, de acordo com a necessidade da planta, conforme Matias et al. (2015). Portanto, a krigagem configura-se como uma ferramenta essencial para se estabelecer o manejo de maior proximidade para cada nutriente (Matias et al., 2015).

Os mapas de variabilidade espacial da produtividade e atributos químicos do solo são apresentados na Figura 2.

Todos os atributos químicos obtiveram correlações baixas e não significativas em relação à produtividade média. A correlação negativa entre a produtividade média e o pH que apresenta correlação quase nula evidencia uma leve posição inversamente proporcional à produtividade da cultura,

Quadro 2 - Modelos teóricos dos semivariogramas ajustados para as variáveis produtividade média, fósforo (P); potássio (K); capacidade de trocas catiônicas (CTC) potencial hidrogeniônico (PH) e matéria orgânica (MO)

\begin{tabular}{cccccccc}
\hline & \multicolumn{7}{c}{ Geoestatística } \\
\cline { 2 - 7 } Parâmetros & Modelo & Co & Co+C1 & A & $\mathbf{R}^{2}$ & RSS & IDE \\
\hline Produtividade & Exponencial & 0,2737 & 0,5774 & 481.00 & 0,959 & $1,793.10^{-3}$ & Moderada \\
Fósforo & Exponencial & 5190,00 & 10390,00 & 117,70 & 0,971 & $2,80.10^{6}$ & Moderada \\
Potássio & Exponencial & 904,000 & 2104,00 & 567,00 & 0,979 & 5872 & Moderada \\
Capacidade de Troca & Exponencial & 0,12800 & 0,85500 & 63,00 & 0,882 & 0,0115 & Forte \\
$\begin{array}{c}\text { Catiônica } \\
\text { Potencial Hidrogeniônico } \\
\text { Matéria Orgânica }\end{array}$ & Gaussiano & 0,00267 & 0,02054 & 100,00 & 0,810 & $2,367.10^{-5}$ & Forte \\
\hline
\end{tabular}

Co - efeito pepita; Co + C1 - patamar; a - alcance (m); IDE = Co/ (Co+ C1) - Índice de Dependência Espacial (\%). IDE - Índice de Dependência Espacial baixo para IDE $<25 \%$, moderada para $25 \%<$ IDE $<75 \%$ e forte para IDE $>75 \%$ (Zimback, 2001). 

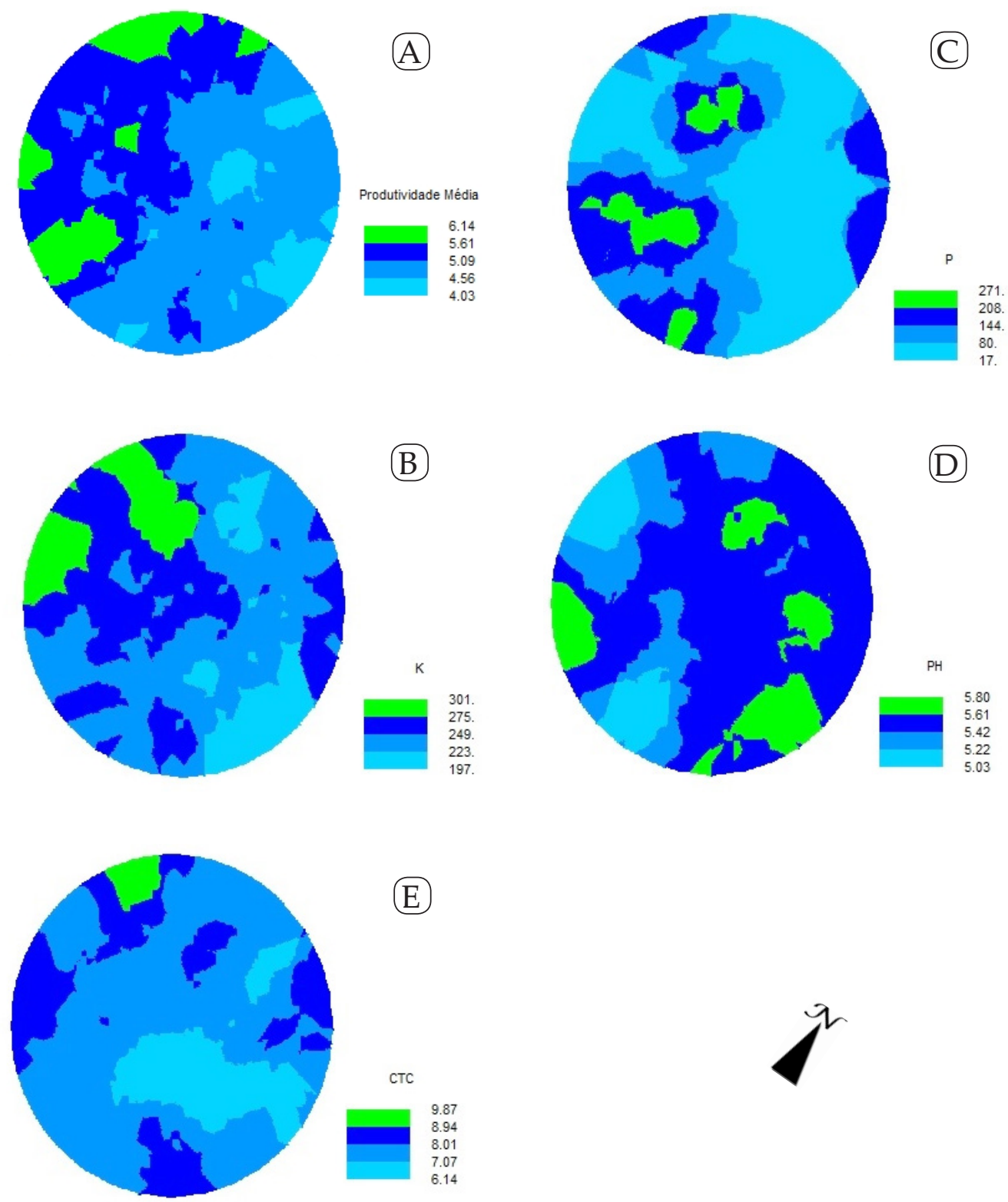

Figura 2 - Mapas da distribuição espacial A - produtividade média (t ha-1); B - Fósforo (mg dm³); C - Potássio (mg dm³); D potencial hidrogeniônico (pH); E - Capacidade de Trocas Catiônicas (cmolc dm-3).

o que pode estar ocorrendo na área uma neutralização excessiva de algumas regiões dentro da área, restringindo a absorção de nutrientes pelas plantas, comprometendo a produtividade (Mattioni et al., 2013). Os autores ainda ressaltam que a cultura da soja é afetada por inúmeros fatores durante o ciclo vegetativo e que afetam diretamente a produtividade da cultura dificultando o isolamento de alguns fatores na realização deste tipo de trabalho.
Apesar de não apresentar correlações significativas, os mapas de produtividade e atributos químicos expõem similaridade na localização dos maiores e menores valores, com proporções de área com maiores valores na região noroeste dos mapas. No entanto a geoespacialização da produtividade apresenta-se com distribuição contrária à distribuição dos valores de $\mathrm{pH}$, ou seja, onde se observam maiores produtividade, menores as os valores de $\mathrm{pH}$ na área, e vice-versa. 
Analisando a distribuição espacial da produtividade e os atributos químicos P, K, e CTC destaca-se similaridade, onde se apresentam maiores valores de produtividade $\left(5,61\right.$ a $\left.6,14 \mathrm{~T}^{\mathrm{h}} \mathrm{h}^{-1}\right)$ encontram-se valores considerados muito alto de $\mathrm{P}\left(>80 \mathrm{mg} \mathrm{dm}^{-3}\right)$ e K (> 234,6 $\mathrm{mg} \mathrm{dm}^{-3}$ ) (Raij et al., 1997).

Na Figura 3 são apresentados os mapas de classificação categórica da produtividade e atributos químicos do solo utilizando 3 classes de divisão, a fim de buscar uma melhor representação da distribuição dos valores na área de estudo. Pode-se observar que para produtividade a classe considerada alta foi predominante na região noroeste do mapa apresentando 8,217 ha.

Os atributos químicos também exibiram concentrações de classes altas na região noroeste dos mapas, exibindo 6,036 ha de alto teor de $\mathrm{P}, 6,508$ ha de alto teor de K, e 4,769 ha de alto teor de CTC, entretanto, a região noroeste do mapa de $\mathrm{pH}$ a classe predominante foi baixa, 7,47 ha. Por meio dos mapas de classes e distribuição espacial é possível aprimorar as zonas de manejo aos atributos químicos (exceto $\mathrm{pH})$, a fim de resultar no aumento de locais da produtividade que apresentaram classe baixa.
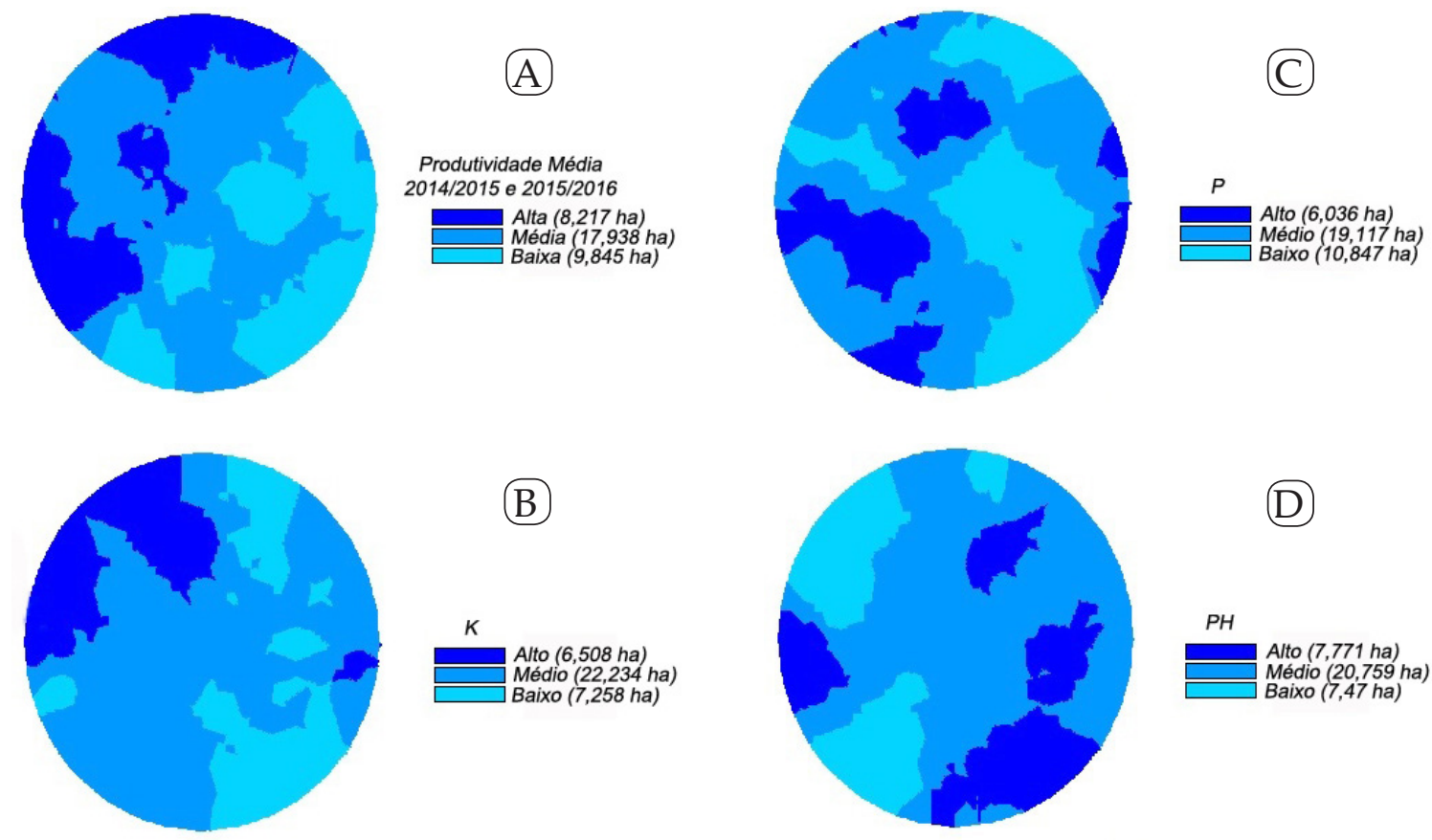

(D)

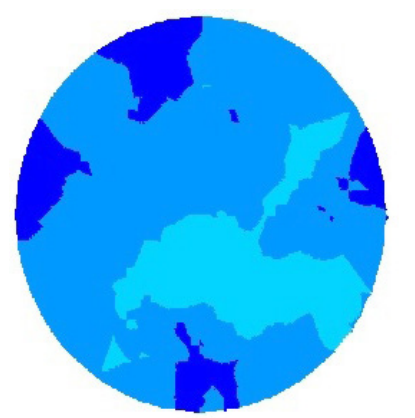

(E)
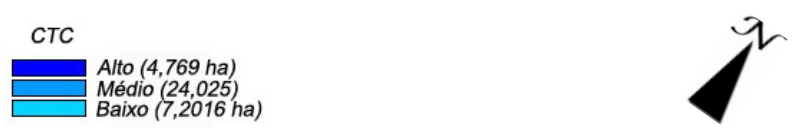

Figura 3 - Mapas da classificação categórica A - produtividade média; B - Fósforo; C - Potássio; D - potencial hidrogeniônico; E - Capacidade de Trocas Catiônicas. 


\section{CONCLUSÕES}

As definições de zonas de manejo por meio de mapas de classe foram satisfatórias para a produtividade média e os atributos químicos avaliados;
As áreas de baixa e média produtividade correspondem a 27,78 ha, predomina-se nas regiões central, sudeste e nordeste do mapa.

Mesmo não apresentado correlação significativa a produtividade e atributos químicos apresentaram maiores valores na região noroeste do mapa.

\section{REFERÊNCIAS}

Alves, S.M.F.; Queiroz, D.M.; Alcântara, G.R. \& Reis, E.F. (2014) - Variabilidade espacial de atributos físicoquímicos do solo usando técnicas de análise de componentes principais e geoestatistica. Bioscience Journal, vol. 30 , sup. 1 , p. $22-30$.

Aquino, R.E.; Campos, M.C.C.; Júnior, J.M.; Oliveira, I.A.; Mantovaneli, B.C. \& Soares, M.D.R. (2014) Geoestatística na avaliação dos atributos físicos em Latossolo sob floresta nativa e pastagem na região de Manicoré, amazonas. Revista Brasileira de Ciências do Solo, vol. 38, n. 2, p. 397-406. http://dx.doi.org/10.1590/ S0100-06832014000200004

Artur, A.G.; Oliveira, D.P.; Costa, M.C.G.; Romero, R.E.; Silva, M.V.C. \& Ferreira, T.O. (2014) - Variabilidade espacial dos atributos químicos do solo, associada ao microrrelevo. Revista Brasileira de Engenharia Agrícola e Ambiental, vol. 18, n. 2, p. 141-149. http://dx.doi.org/10.1590/S1415-43662014000200003

Bottega, E.L.; Pinto, F.A.C.; Queiroz, D.M.; Santos, N.T. \& Souza, C.M.A. (2013b) - Variabilidade espacial e temporal da produtividade de soja no Cerrado brasileiro. Revista Agrarian, vol. 6, n. 20, p.167-177.

Bottega, E.L.; Queiroz, D.M.; Pinto, F.A.C. \& Souza, C.M.A. (2013a) - Variabilidade espacial de atributos do solo em sistema de semeadura direta com rotação de culturas no cerrado brasileiro. Revista Ciência Agronômica, vol. 44, n. 1, p. 1-9.

Cherubin, M.R.; Santi, A.L.; Eitelwein, M.T.; Amado, T.J.C.; Simon, D.H. \& Damian, J.M. (2015) - Dimensão da malha amostral para caracterização da variabilidade espacial de fósforo e potássio em Latossolo Vermelho. Pesquisa Agropecuária Brasileira, vol. 50, n. 2, p. 168-177. http://dx.doi.org/10.1590/\$0100-204X2015000200009

Donagema, G.K.; Campos, D.V.B.; Calderano, S.B.; Teixeira, W.G. \& Viana, J.H. (2011) - Manual de métodos de análise de solos. Rio de Janeiro: Embrapa Solos, 230 p.

EMBRAPA (2013) - Sistema brasileiro de classificação de solos. 3.ed. Rio de Janeiro: Embrapa Solos. 353p.

Matias, S.S.R.; Baptistel, A.C.; Nóbrega, J.C.A.; Andrade, F.R. \& Silva, J.B.L. (2015) - Variabilidade espacial dos atributos do solo em duas áreas de manejo convencional no Cerrado piauiense. Amazonian Journal of Agricultural and Environmental Sciences, vol. 58, n. 2, p. 217-227. http://dx.doi.org/10.4322/rca.1687

Mattioni, N.M.; Schuch, L.O.B. \& Villela, F.A. (2013) - Variabilidade espacial e efeito de atributos químicos de um Latossolo na população de plantas e produtividade da cultura da soja. Revista da Faculdade de Zootecnia, Veterinária e Agronomia, vol. 19, n. 1, p. 20-32.

Miqueloni, D.P.; Gianello, E.M.; Bueno, C.R.P.; Silva, P.C.M.; Mesquita, F.O. \& Costa, T.K.G. (2015) Variabilidade espacial de atributos e perda de solo na definição de zonas de manejo. Pesquisa Agropecuária Tropical, vol. 45, n. 1, p. 18-28. http://dx.doi.org/10.1590/1983-40632015v4528029

Montanari, R.; Panachuki, E.; Lovera, L.H.; Correa, A.R.; Oliveira, I.S.; Queiroz, H.A. \& Tomaz, P.K. (2015) - Variabilidade espacial da produtividade de sorgo e de atributos do solo na região do Ecótono Cerrado-Pantanal, MS. Revista Brasileira Ciências do Solo, vol. 39, n. 2, p. 385-396. http://dx.doi. org/10.1590/01000683rbcs20140215

Montgomery, D.C. \& Runger, G.C. (2009) - Estatística aplicada e probabilidade para engenheiros. ed. 4, 493 p. Rio de Janeiro, editora LTC.

Nascimento, P.S.; Silva, J.A.; Costa, B.R.S.; Bassoi, L.H.M.; Mesquita, F.O. \& Costa, T.K.G. (2014) - Zonas homogêneas de atributos do solo para o manejo de irrigação em pomar de videira. Revista Brasileira Ciências do Solo, vol. 38, n. 4, p.1101-1113. http://dx.doi.org/10.1590/S0100-06832014000400006 
Pimentel-Gomes, F. \& Garcia, C.H. (2002) - Estatística aplicada a experimentos agronômicos e florestais: exposição com exemplos e orientações pra uso de aplicativos. Piracicaba: Fealq. 309 p.

Raij, B.V.; Cantarella, H.; Quaggio, J.A. \& Furlani, A.M.C. (1997) - Recomendações de adubação e calagem para o estado de São Paulo. 2.ed.atual. Campinas-SP: Instituto Agronômico/Fundação IAC, 1997. 285p. (Boletim Técnico, 100).

Shapiro, S.S. \& Wilk, M.B. (1965) - An analysis of variance test for normality: complete samples. Biometrika, vol. 52, n. 3-4, p. 591-611. https://doi.org/10.2307/2333709

Souza, Z.M.; Marques Júnior, J. \& Pereira, G.T. (2009) - Geoestatística e atributos do solo em áreas cultivadas com cana-de-açúcar. Ciência Rural, vol. 40, n. 1, p. 48-56. http://dx.doi.org/10.1590/\$0103-84782009005000243

Weber, E.A. (2001) - Armazenagem agrícola. 2 ed. Guaíba: Agropecuária. 396 p.

Zimback, C.R.L. (2001) - Análise espacial de atributos químicos de solos para fins de mapeamento da fertilidade do solo. 2001. 114 f. Tese (Livre-Docência) - Faculdade de Ciências Agronômicas, Universidade Estadual Paulista, Botucatu. 